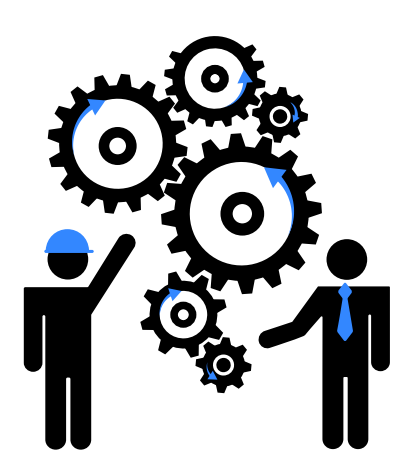

\title{
DESARROLLO A NIVEL FUNCIONAL DE UN PUENTE TRANSPORTABLE Y TELEOPERADO
}

\author{
FUNCTIONAL DEVELOPMENT OF TRANSPORTABLE AND TE- \\ LEOPERATE BRIDGE
}

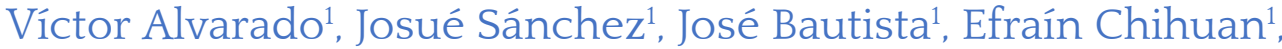 William Buendía ${ }^{1}$, Roberto Cuazitil ${ }^{1}$, Celso De La Cruz ${ }^{2}$}

1 Escuela Académico Profesional de Ingeniería Mecatrónica, Universidad Continental E-mail: 46927485@continental.edu.pe

2 Unidad de Investigación de la Facultad de Ingeniería, Universidad Continental E-mail: cdelacruz@continental.edu.pe

\section{Resumen}

Las vías de comunicación terrestre en el Perú son bastante accidentadas. Debido a las abundantes lluvias que se presentan en la sierra del Perú y ocasionan la crecida de los ríos y estos a su vez los desastres naturales, estas vías terrestres se dañan y aíslan comunidades y ciudades, hecho que perjudica a la economía de estas poblaciones y, por ende, a la economía del país. La presente investigación tiene por objetivo proponer una alternativa de solución para restablecer la comunicación de manera temporal: desarrollar a nivel funcional un puente transportable y teleoperado. La teleoperación es el mecanismo mediante el cual se evita la exposición al riesgo de personas. Además, se detallan los materiales utilizados en la construcción e implementación de un prototipo a escala, mediante la plataforma de Arduino para el control y los módulos de bluetooth para la teleoperación del mismo. La potencia la generan unos servomotores con la capacidad necesaria para realizar los desplazamientos del mecanismo. Las pruebas experimentales del prototipo muestran la viabilidad funcional de esta solución, que servirá de base para un prototipo a escala normal.

Palabras clave: Puente transportable; teleoperación; Arduino; Bluetooth, desastres naturales.

\begin{abstract}
Roads of terrestrial communication in Peru are quite uneven. Due to the abundant rains that occur in the highland of Peru, which cause increasing of rivers and as consequence natural disasters these roads are damaged and isolate communities and cities, a fact that harms the economy of these populations and, therefore, to the country's economy. This research aims to achieve an alternative solution in order to reestablish a temporal communication: to develop a transportable and teleoperated bridge at functional level. Teleoperation is the mechanism by which exposure has avoided the risk of people. In addition, the materials used in the construction and implementation of a prototype to scale are detailed, through the Arduino platform for the control and the bluetooth modules for teleoperation of the same. The power is generated by servomotors with the necessary capacity to carry out the movements of the mechanism. The experimental tests of the prototype show the functional viability of this solution, which will serve as the basis for a prototype on a normal scale.
\end{abstract}

Keywords: Transportable bridge; teleoperation; Arduino; Bluetooth; natural disaster. 


\section{INTRODUCCIÓN}

La necesidad que motivó la presente investigación es el requerimiento del diseño y construcción de un puente estructural con control automático y de fácil transporte para zonas en emergencia a fin de evacuar a personas y transportar alimentos perecibles de primera necesidad durante desastres naturales u algún otro tipo de emergencias que se puedan suscitar.

La necesidad de reestablecer la comunicación terrestre, interrumpida a causa de los huaycos, el desborde de ríos y las carreteras colapsadas, es de prioridad en épocas específicas y esto se logrará mediante puentes temporales de auxilio rápido, que sean transportables a las zonas de emergencia en cualquier tipo de desastre natural.

En la literatura se encuentran propuestas de puentes peatonales y de vehículos modulares de armado en un tiempo menor para atender las urgencias de comunicación en los lugares aislados por desastres naturales [1], [2]. Sin embargo, el armado de estos puentes modulares no es lo suficientemente rápido.

El trabajo [3] propone diseñar un puente de vigas hinchables, ligero, modular y portátil para el paso en superficie de vehículos, que utilice como elemento básico estructural para aguantar las cargas vigas hinchadas con aire a baja presión y fabricadas con tejidos compuestos de altas prestaciones. La velocidad de armado de este tipo de puentes es muy rápida. Otros puentes transportables de rápido armado se han utilizado en guerras [4], estos puentes son flotantes, tienen una gran capacidad de carga para vehículos pesados y permiten transportar vehículos militares de una orilla del río hacia la otra orilla. Los puentes son transportados en camiones y se arman utilizando actuadores hidráulicos. También existen puentes tipo tijera [5], que tienen dos cuerpos y se despliegan utilizando actuadores hidráulicos; su armado es rápido y se pueden transportar en camiones.

Los puentes de rápido armado, presentados anteriormente, son ensamblados en el sitio del desastre natural por operadores que tienen que exponerse a posibles deslizamientos de tierra. En el presente trabajo se propone un puente tipo tijera de armado teleoperado a fin de proteger al operario, quien estará comandando el armado desde un lugar seguro.

El diseño del sistema mecánico se hizo con el software SolidWorks ${ }^{\circledR}$; para el sistema de control del prototipo, se utilizó el software y módulo de la plataforma de Arduino; en los sistemas de potencia se optó por los motorreductores para la parte de tracción de las orugas y para la instalación del puente se utilizaron unos servomotores por su capacidad y control preciso del giro; para la parte de comunicación, se utilizaron módulos de bluetooth y un celular con sistema operativo Android. Asimismo, se realizaron cálculos matemáticos para obtener los torques y luego elegir los actuadores óptimos para este sistema.

Las pruebas del prototipo permitieron verificar la funcionalidad del puente transportable de armado con teleoperación.

\section{BÚSQUEDA DE SOLUCIONES}

\section{Teleoperación}

Dado que el sistema del puente tiene que trabajar en zonas de emergencia, es necesaria manejarlo mediante teleoperación para evitar que el operador sufra accidentes en el manejo del equipo. Para esto se plantean las siguientes tecnologías como posibles soluciones:

\section{Wifi}

Es un mecanismo de conexión de dispositivos electrónicos de forma inalámbrica. Los dispositivos habilitados con wifi (como una computadora personal, un televisor inteligente, una videoconsola, un teléfono inteligente o un reproductor de música) pueden conectarse a Internet a través de un punto de acceso de red inalámbrica. Dicho punto de acceso tiene un alcance de unos veinte metros en interiores, distancia que es mayor al aire libre.

Entre sus ventajas están facilitar información en tiempo real en cualquier lugar para todo usuario de la red y permitir el uso múltiple de la red por varios usuarios al mismo tiempo. Sus desventajas son el bloqueo de la señal o interferencias y la vulnerabilidad.

\section{Bluetooth}

Bluetooth es una especificación industrial para Redes Inalámbricas de Área Personal (WPAN), posibilita la transmisión de voz y datos entre diferentes dispositivos mediante un enlace por radiofrecuencia en la banda ISM (Industrial, Scientific and Medical) de los $2.4 \mathrm{GHz}$. Los principales objetivos que se pretenden conseguir con esta norma son:

- Facilitar las comunicaciones entre equipos móviles.

- Eliminar los cables y conectores entre estos.

- Ofrecer la posibilidad de crear pequeñas redes 
inalámbricas y facilitar la sincronización de datos entre equipos personales.

- Los dispositivos que utilizan esta tecnología con mayor frecuencia pertenecen a los sectores de telecomunicaciones y la informática personal, como PDA, teléfonos móviles, computadoras portátiles, ordenadores personales, impresoras o cámaras digitales.

\section{Ventajas}

- Es muy fácil crear una red inalámbrica entre varios dispositivos para sincronizar e intercambiar información.

- Si uno no sabe mucho sobre tecnología, no es difícil aprender a utilizar este protocolo por primera vez.

- No quita demasiada autonomía a los gadgets que utilizan Bluetooth debido a que se manejan unos pocos mili-Watt.

- Las velocidades de las últimas dos versiones (3.0 y 4.o) son altas (de $24 \mathrm{MB} / \mathrm{s}$ ambas).

\section{Desventajas}

- La seguridad es un factor desfavorable del Bluetooth. En la actualidad se han presentado mejoras, sobre todo en los celulares; algunos años atrás el protocolo podía resultar inseguro debido a una configuración incorrecta, asimismo, era vulnerable a la pérdida de información.

- El reducido alcance del protocolo para intercambiar información debido a la baja potencia que maneja; esta es una gran desventaja ya que va desde $1 \mathrm{~m}$ en la clase 3 hasta $30 \mathrm{~m}$ en la clase 1.

\section{Radio frecuencia}

Es la técnica que permite el gobierno de un objeto a distancia y de manera inalámbrica mediante una emisora de control remoto.

En el radiocontrol entran en juego tres técnicas fundamentales: a) la electrónica, que transforma los comandos dados en ondas de radio en el transmisor y a la inversa en el receptor; b) la electricidad, encargada de proporcionar la energía necesaria a los dispositivos tanto al comando (o transmisor) como al receptor, y c) la mecánica, que mueve los accionadores (o servos) que dan las señales eléctricas demoduladas o decodificadas en movimiento mecánico.

Existen todo tipo de vehículos de modelismo dirigidos por radiocontrol, los más populares son los co- ches, los aviones, los barcos, los helicópteros y los submarinos.

\section{Ventajas}

- Bajo consumo

- Distancia de uso

\section{Desventajas}

- Interferencias de radios

\section{Sensor de altura}

Se propone utilizar un sensor de altura para detectar el nivel del agua del río; de tal manera que cuando el nivel se incremente demasiado, este emita una alarma para que los vehículos dejen de transitar por el puente y se inicie, mediante teleoperación, las operaciones de recojo del mismo. Entre las opciones para detectar el nivel del río, se encuentran:

\section{Válvula de flotador}

Es un mecanismo de retroalimentación mecánico que regula el nivel del fluido mediante el uso de un flotador para mover un interruptor, de tal manera que ante un mayor nivel de líquido se fuerza a cerrar el interruptor, mientras que en un menor nivel de líquido se fuerza la apertura del interruptor.

\section{Sensor de nivel ultrasónico sin contacto}

Estos sensores incorporan un procesador de señal analógica, un microprocesador, decimal codificado en binario (BCD), switches de rango y un circuito de salida del controlador. Transmiten los impulsos a una puerta de señal de la ruta del microprocesador a través del procesador de la señal analógica del sensor, que envía un haz ultrasónico a la superficie del líquido. El sensor de nivel detecta el eco de la superficie y la envía de vuelta al microprocesador para una representación digital de la distancia entre el sensor y el nivel de la superficie. A través de una actualización constante de las señales recibidas, el microprocesador calcula los valores promedios para medir el nivel de líquido.

\section{Sensor de nivel ultrasónico de contacto}

Es un dispositivo ultrasónico de baja energía dentro de los sensores de nivel de líquido en un punto determinado. Consta de un sensor montado y un amplificador integrado de estado sólido, los sensores 
ultrasónicos de contacto no tienen partes móviles y no requieren calibración. Típicamente, están equipados con bloques de terminales para la conexión de una fuente de alimentación y dispositivos de control externos. La señal ultrasónica atraviesa un hueco de $12 \mathrm{~mm}$ en el sensor y controla los interruptores de relé cuando la brecha contiene líquido. El nivel de detección está en el medio a lo largo del espacio donde los sensores están montados en horizontal. En la parte superior, por sensores montados verticalmente, a medida que el líquido cae por debajo de este nivel, la señal ultrasónica atenúa y finalmente conmuta el relé a su estado anterior.

\section{Sensor de nivel por capacitancia}

$\mathrm{Al}$ igual que los sensores ultrasónicos, los sensores por capacitancia pueden manejar la medición de nivel puntual o continuo; usan una sonda para monitorear los cambios de nivel de líquido en el tanque, acondicionando electrónicamente la salida a valores capacitivos y resistivos, que se convierten en señales analógicas. La sonda y el recipiente equivaldrán a las dos placas de un capacitor y el líquido, al medio dieléctrico. Debido a que la señal emana solo cuando hay cambios de nivel, la acumulación de material en la sonda no tiene efecto. Los recipientes de fluido no conductor pueden indicar sondas dobles o una banda conductora externa.

La sonda, que puede ser rígida o flexible, normalmente usa alambre conductor con aislamiento de OPTE. El uso de acero inoxidable como material de la sonda ofrece la sensibilidad adicional que se necesita para medir líquidos que son no conductores, granulares o de propiedades dieléctricas bajas (constante dieléctrica menor de 4). Se deben usar sondas flexibles cuando no hay suficiente espacio libre para una sonda rígida o en aplicaciones que exigen longitudes muy grandes. Las sondas rígidas ofrecen una estabilidad más alta, especialmente en sistemas turbulentos, donde la oscilación de la sonda puede causar fluctuaciones en la señal.

\section{Elección de la solución}

Debido a que el proyecto tiene que trabajar en zonas de emergencia, sugiere un alto riesgo para las personas, por lo cual se utilizaría la opción (A) Teleoperación, mediante el módulo WIFI ESP8266, que es compatible con Arduino; estableciendo un medio de comunicación entre el sistema de control y el puente y controlado con un celular Galaxy S8.
Las ventajas que ofrece son:

\section{Viabilidad-tecnológica}

Gran velocidad de transmisión de datos según la frecuencia que se trabaje 2,4 Ghz; mayor alcance de transmisión en promedio de los $100 \mathrm{~m}$ a campo abierto, seguridad en la conexión debido a su encriptación de datos (WPS2).

\section{Viabilidad-técnica}

Tiene una interfaz de fácil programación e instalación.

Para el prototipo a escala, se utilizará el módulo Bluetooth dado que las distancias entre el sistema de teleoperación y el puente en estas pruebas serán cortas.

El puente requiere de sensores para medir el nivel del río y dar aviso del riesgo para sus transeúntes; para tal caso, se utilizará el sensor ultrasónico sin contacto. A diferencia de otros sensores, presenta cualidades de medición que no necesitan estar en contacto con el agua para brindarnos los datos medidos, además se puede utilizar a canal abierto.

El sistema de seguridad se implementará con la «Alarma Audio-Visual», que cuenta con una sirena (buzzer) y un diodo LED para dar aviso de emergencia.

\section{DESCRIPCIÓN DE LA SOLUCIÓN}

En la Figura 1 se muestra el diseño del puente tipo tijera, en Solidworks, desplegado en un 50 \%; solo se movió el primer eje de la base en $90^{\circ}$. El puente totalmente desplegado se muestra en la Figura 2; para llegar a esta posición, el segundo eje de la base tiene un movimiento de $90^{\circ}$ y realiza un giro total de $180^{\circ}$, y el eje que une a las dos partes del puente (tercer eje) tiene un movimiento de $180^{\circ}$. Una vez desplegado totalmente, el puente se desacopla del carro y queda instalado y listo para que circulen los vehículos.

El primer eje cumple la función de montary desmontar el puente sobre el carro. Debido a que el puente se separa del carro, se utilizan dos microcontroladores: el primero montado en el carro para manejar los servos del primer y segundo ejes, y el segundo montado en el puente para manejar el motor reductor del tercer eje y la sirena de alarma. Ambos microcontroladores se comunican por módulos Bluetooth. 


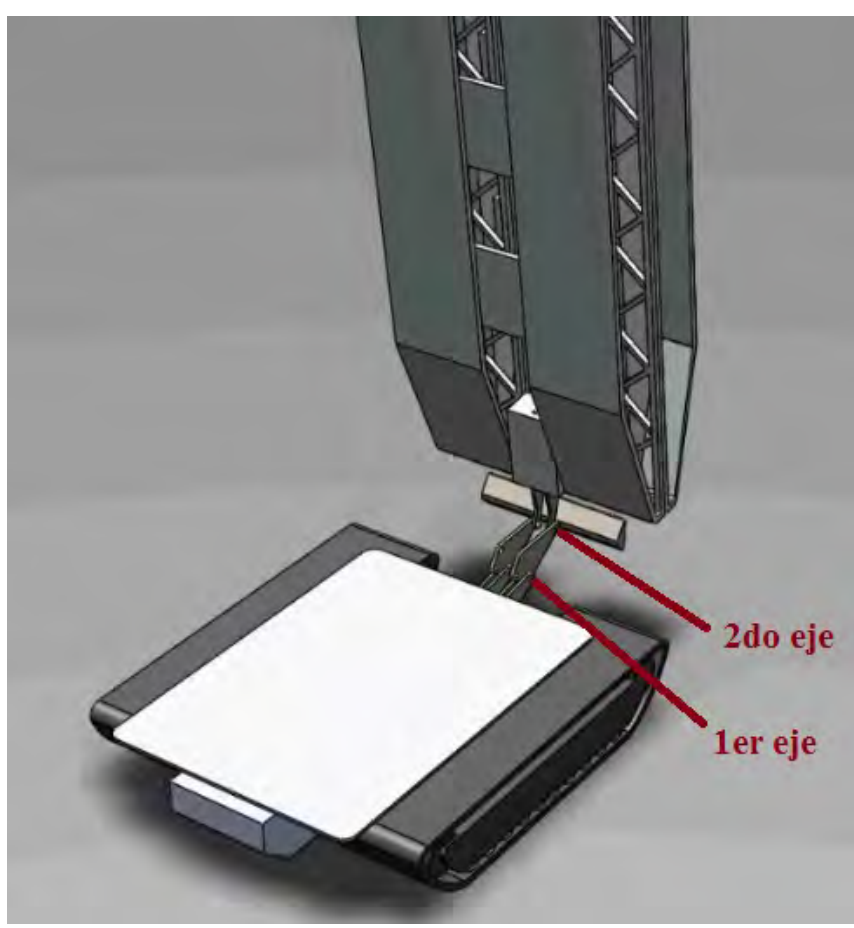

Figura 1. Diseño del puente en Solidworks. Puente desplegado a un $50 \%$.

Fuente: Elaboración propia.

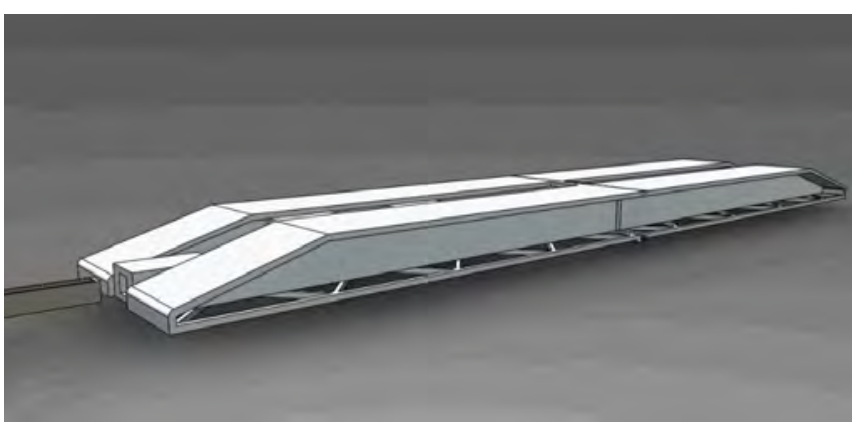

Figura 2. Puente desplegado al $100 \%$.

Fuente: Elaboración propia.

Cálculo del torque de los servomotores para el despliegue del puente

Para el caso del primer eje del puente, donde la fuerza necesaria es el esfuerzo que tiene que cargar, es decir la masa de todo el puente $(0.528 \mathrm{~kg})$, la longitud de cada mitad es de $23,5 \mathrm{~cm}$. Se requiere el mayor toque en el primer eje cuando el puente está totalmente replegado.

Cálculo del torque máximo del primer eje: cuando se inicia el despliegue del puente.

Torques producidos:

Torque $1=($ Fuerza $\mathrm{x}$ distancia al centro de gravedad $)$

Torque $1=(0,528 \mathrm{~kg} \times 23,5 \times 0.5 \mathrm{~cm})$

Torque $1=6,2$ kgf.cm
Para el caso del segundo eje del puente, donde la fuerza necesaria es el esfuerzo que requiere para cargar todo el puente $(0,528 \mathrm{~kg})$, se necesita el mayor toque en el segundo eje cuando el puente está totalmente desplegado; en este caso el centro de gravedad se encuentra en la mitad del puente.

Cálculo del torque máximo del segundo eje, cuando se inicia el repliegue del puente:

Torques producidos:

Torque 2 = (Fuerza $x$ distancia al centro de gravedad $)$

Torque $2=(0,528 \mathrm{~kg} \times 23,5 \mathrm{~cm})$

Torque $2=12,4$ kgf.cm

Para el caso del tercer eje del puente, donde la fuerza necesaria es el esfuerzo que requiere para cargar la mitad del puente $(0,239 \mathrm{~kg})$, la longitud de cada mitad es de $23,5 \mathrm{~cm}$.

Cálculo del torque máximo del tercer eje: cuando la segunda mitad del puente se encuentra horizontal.

Torques producidos:

Torque $3=($ fuerza $\mathrm{x}$ distancia al centro de gravedad $)$

Torque $3=(0,239 \mathrm{~kg} \times 23,5 \times 0.5 \mathrm{~cm})$

Torque $3=2,8 \mathrm{kgf.cm}$

\section{Selección de los servomotores}

El servo Futaba S5050-Digital Micro Servo HiSpeed/Torque w/Micro Plug:

Datasheet:

Torque:

$4.8 \mathrm{~V}: 211.00 \mathrm{oz}-\mathrm{in}(15.19 \mathrm{~kg}-\mathrm{cm})$

6.o V: 264.00 oz-in (19.01 kg-cm)

Running Current: 650mA

Tiene la responsabilidad del mayor torque, es decir del segundo eje. El torque requerido es inferior por lo que el sistema funciona normalmente.

El servo MG996R High Torque-Metal Gear Dual Ball Bearing Servo

Datasheet:

Torque:

$9.4 \mathrm{kgf} \cdot \mathrm{cm}(4.8 \mathrm{~V})$,

$11 \mathrm{kgf} \cdot \mathrm{cm}(6 \mathrm{~V})$

Running Current: 50omA

El torque es menor que los primeros servos, por lo tanto, se utilizará para el primer eje. El torque requerido es inferior por lo que el sistema funciona normalmente. El tercer eje será movido por un mo- 
torreductor de 6 voltios, de torque de $2 \mathrm{~kg}$ - $\mathrm{cm}$. Sin embargo, para aumentar el torque se utiliza un juego de poleas, se incrementa el torque en 3.7 veces.

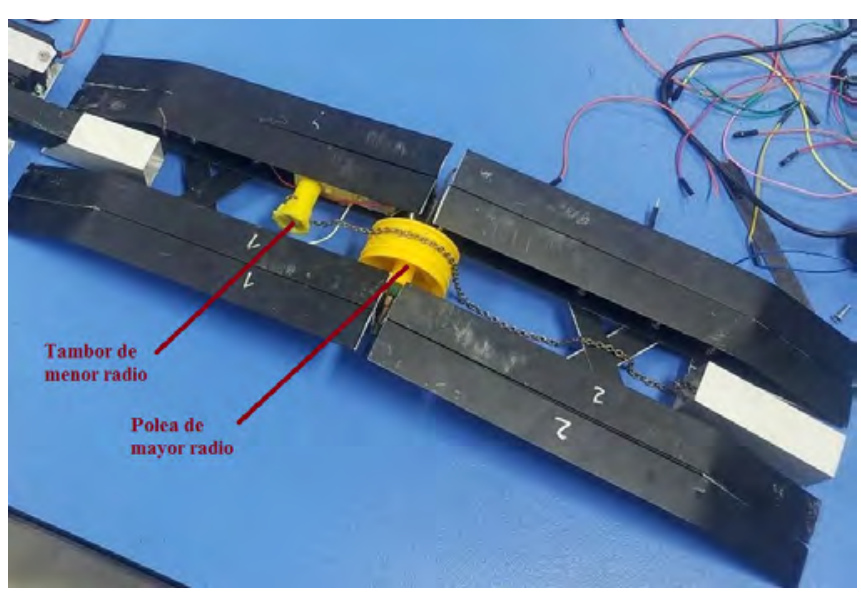

Figura 3. Juego de poleas para incrementar el torque el tercer eje.

\section{Cálculo del consumo de corriente del sistema}

En la Tabla 1 se observa el consumo de corriente del sistema montado en el carro, se consideran las corrientes nominales de cada componente; el consumo total es de 2.47 amperios. La Tabla 2 muestra el consumo de corriente del sistema montado en el puente, que consume 0.159 amperios.

Tabla 1. Consumo de corriente del sistema en el carro

\begin{tabular}{|c|c|c|c|c|}
\hline Îtem & $\begin{array}{c}\text { Componente } \\
\text { electrónico }\end{array}$ & Cantidad & $\begin{array}{c}\text { Consumo } \\
(\mathbf{m A})\end{array}$ & $\begin{array}{c}\text { Consumo } \\
\text { Total } \\
(\mathbf{m A})\end{array}$ \\
\hline 1 & Arduino Mega & 01 & 90 & 90 \\
\hline 2 & $\begin{array}{c}\text { Servo Futaba } \\
\text { S5050 }\end{array}$ & 02 & 650 & 1300 \\
\hline 3 & Servo MG996R & 02 & 500 & 1000 \\
\hline 4 & Bluetooth & 02 & 40 & 80 \\
\hline & & & Total & $\mathbf{2 4 7 0}$ \\
\hline
\end{tabular}

Tabla 2. Consumo de corriente del sistema en el puente

\begin{tabular}{|c|c|c|c|c|}
\hline Ítem & $\begin{array}{c}\text { Componente } \\
\text { electrónico }\end{array}$ & Cantidad & $\begin{array}{c}\text { Consumo } \\
(\mathbf{m A})\end{array}$ & $\begin{array}{c}\text { Consumo } \\
\text { Total } \\
(\mathbf{m A})\end{array}$ \\
\hline 2 & Arduino Nano & 1 & 19 & 19 \\
\hline 5 & $\begin{array}{c}\text { Motorreductor } \\
\text { de 6V }\end{array}$ & 1 & 100 & 100 \\
\hline 6 & Bluetooth & 1 & 40 & 40 \\
\hline & & & Total & 159 \\
\hline
\end{tabular}

\section{IMPLEMENTACIÓN}

\section{Entrada}

\section{Sensor ultrasónico}

Mide la distancia por medio de ultrasonidos, por ello será usado como un sensor que medirá la distancia a la que se encuentra en nivel del agua para el sistema de seguridad. Está colocado estratégicamente dentro de la estructura del puente, casi a la mitad del puente, y, según las condiciones de programación, envía una señal al controlador (ARDUINO MEGA 2560), de tal manera que, si el nivel medido pasa del umbral, se envía un sonido de alerta por intermedio del buzzer.

\section{Software de interface hombre-máquina}

La entrada de datos al sistema es la aplicación desarrollada en el software APP INVENTOR en la que se incluyen botones táctiles apk, estos enviarán señales discretas de manera inalámbrica por medio del bluetooth «MAESTRO» y recibirá dicha señal discreta por medio de un bluetooth «ESCLAVO» conectado al controlador que estos proporcionarán una señal discreta que comandará cada uno de los actuadores según sea la programación para el traslado, colocación y despliegue del puente.

\section{Comunicación}

\section{Bluetooth}

El módulo de comunicación es el bluetooth. Al enviar las diferentes señales discretas provenientes del pulso de las diferentes botoneras táctiles apk, estas serán transmitidas de forma inalámbrica por un celular con sistema operativo Android y serán recibidas por un bluetooth, configurado como «Maestro», colocado en el circuito del carro que también cuenta con un segundo módulo bluetooth, configurado como «esclavo», que envía los datos al arduino nano montado en el puente por medio de otro módulo bluetooth.

\section{Control}

\section{Arduino Mega 2560}

Es una plataforma de código abierto, usada en este proyecto como un sistema de control y procesamiento de señales; tiene como entradas a la aplicación y al bluetooth esclavo y como salidas a los diferentes actuadores, servomotores, que, de acuerdo a las diferentes condiciones que se le dio en el algoritmo lógico con respecto a las entradas, puedan decidir y 
enviar señal discreta a la salida para que pueda accionar los diferentes actuadores según cumplan la condición del algoritmo.

\section{Arduino Nano}

Es una pequeña y completa placa basada en el ATmega328, utilizada en este proyecto con el propósito de cumplir ciertos requerimientos de control para actuadores como: motor DC, buzzer y los leds de acuerdo a ciertas condiciones que se generan en las variables del algoritmo de control procedentes de las entradas como: sensor ultrasónico HC-SRo4, aplicación apk, etc.

\section{Actuadores}

En esta fase del proyecto se utilizaron varios actuadores, tales como, diodos emisores de luz, motores DC, servomotores y un buzzer, los cuales actúan según se cumplan las condiciones del algoritmo de control para el que fue programada.

\section{Programación}

\section{Carro}

En la programación del carro se usaron las librerías «Servo» para el control de los servomotores y el SoftwareSerial para conectar dos bluetooth a un solo arduino.

\section{Puente}

El circuito colocado en el puente debe sincronizarse con el circuito del carro al momento de ensamblar el puente. Por eso se utiliza la librería SoftwareSerial para obtener los datos mediante el módulo bluetooth. Este circuito también debe encargarse de leer los datos del sensor ultrasónico y analizar los datos obtenidos para activar la alarma audiovisual.

\section{Software de interface hombre-máquina}

Para la programación de la aplicación, se usó la plataforma online MIT App Inventor 2, ya que proporciona un sistema de programación con bloques para acelerar el diseño de la aplicación.

En la Figura 4 se muestra la pantalla del aplicativo, la cual brinda las opciones de avanzar, retroceder, girar a la derecha y a la izquierda y parar el carro. También, muestra el estado de conexión del sistema de comunicación y los botones para replegar y desplegar el puente.
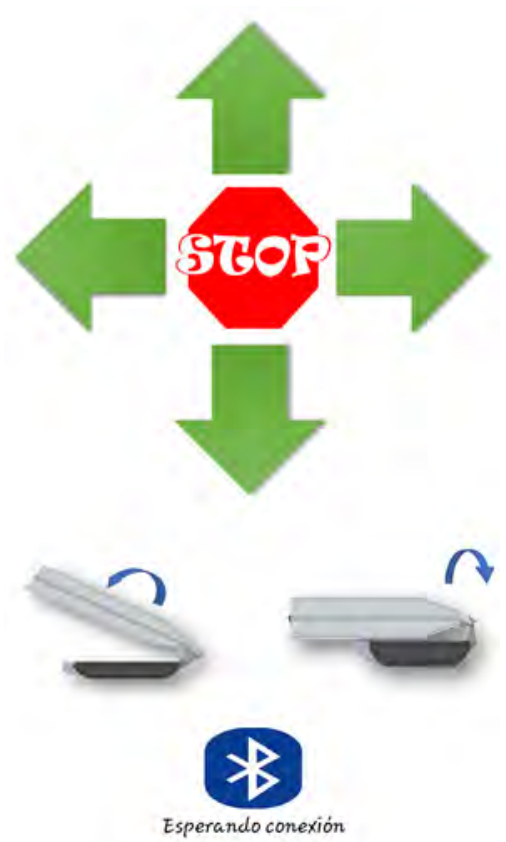

Figura 4. Aplicativo de interface hombre-máquina.

\section{RESULTADOS}

Las pruebas del prototipo se realizaron sobre unas mesas; primero se probó el movimiento del carro con el puente replegado (Figura 5). Utilizando el aplicativo del celular, el carro fue movido hasta el punto que representa la orilla del río, luego, se eligió la opción de despliegue del puente. El puente automáticamente realiza las secuencias de despliegue siguientes: el primer eje se mueve $90^{\circ}$; el segundo eje se mueve $90^{\circ}$ junto a el tercer eje que se mueve $180^{\circ}$. Finalmente, cuando el puente está completamente desplegado, se desacopla el puente del carro (Figura 6).

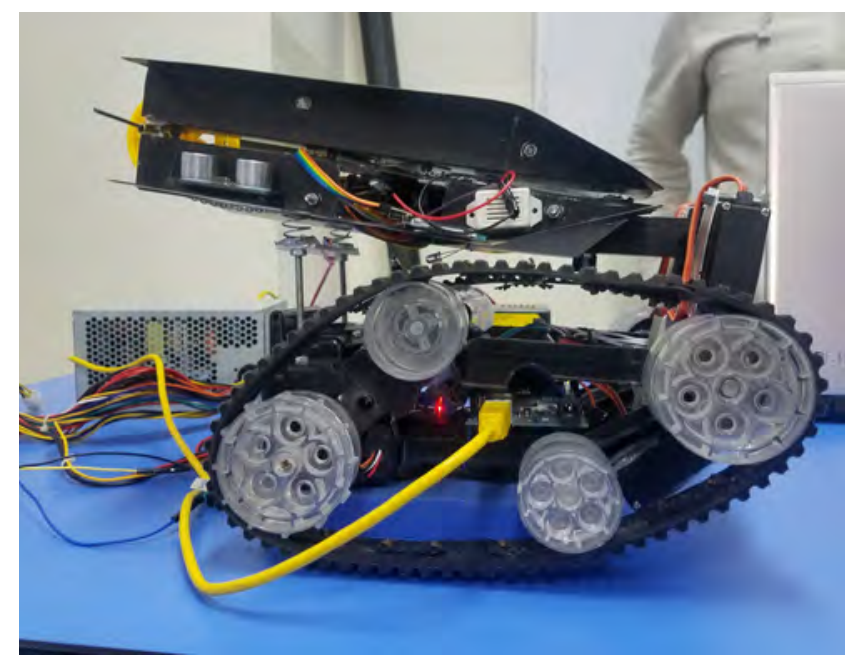

Figura 5. Carro con el puente totalmente replegado. 

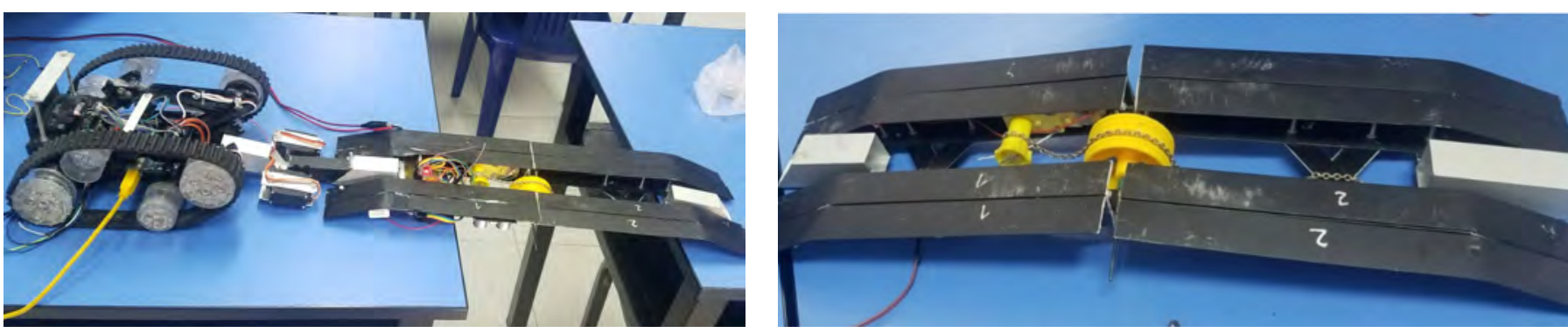

Figura 6. Puente desplegado y desacoplado del carro

A pesar del buen funcionamiento del prototipo, hay algunos puntos por mejorar, como la sincronización en el desplegado del puente, para ello el segundo eje debe girar a la mitad de la velocidad del tercer eje, a fin de evitar esfuerzos adicionales innecesarios en los motores cuando el tercer eje se mueve más rápido de lo necesario. Una mayor velocidad en el tercer eje ocasiona que el centro de masa se aleje del segundo eje y así genera un mayor torque en el segundo eje.

El proceso de replegado del puente es en orden inverso al desplegado.

También se observó que el primer eje cumple la función fundamental de acomodar el puente sobre el carro, tal como se había considerado en el diseño.

\section{VI . CONCLUSIONES}

- El puente móvil para huaycos es viable para situaciones de emergencia como desbordes de ríos, brinda protección al operador y a los vehículos y personas que transitan sobre el puente.

- El control implementado por medio de bluetooth, teléfono celular y microcontroladores Arduino brindó los resultados esperados.

- Los cálculos de los actuadores del sistema mecánico fueron adecuados porque no presentaron trabas, calentamiento o averías en las pruebas.
- El mecanismo con tres ejes es el adecuado para el desplegado y replegado del puente.

- La sincronización del segundo y tercer eje es necesario para evitar sobreesfuerzos en los actuadores.

- Como trabajo futuro se propone la realización del diseño mecatrónico del sistema a escala real.

\section{REFERENCIAS}

[1] BIANCONI, M.S., O. APANGO y H. RAMÍREZ. Puente portátil peatonal desmontable para emergencias. Legado de Arquitectura y Diseño, núm.18, 2015.

[2] APANGO, O. Diseño y evaluación de sistemas estructurales modulares para puentes vehiculares. [Tesis doctoral]. Universidad Autónoma del Estado de México, 2012.

[3] ESTRUCH, C. Nuevo concepto de Puente de vigas hinchables ligero, modular y portátil. [Tesis doctoral]. Universidad Politécnica de Catalunya, 2016.

[4] GARCÍA, C., y A. SORROZA. Armas para una III guerra del Golfo. Real Instituto Elcano de Estudios Internacionales y Estratégicos.

[5] WIKIPEDIA. M6o AVLB. Actualizado el 25 de junio de 2017 [Fecha de consulta 22 de noviembre de 2017]. Disponible en: https://en.wikipedia.org/wiki/M6o_AVLB. 